\title{
CARACTERIZAÇÃO DE POLIÓIS INDUSTRIAIS POR MALDI-TOF
}

\author{
J. A. DA SILVA ${ }^{1}$, L. NAGY² e L. G. AGUIAR ${ }^{1}$ \\ ${ }^{1}$ Universidade de São Paulo, Departamento de Engenharia Química \\ ${ }^{2}$ University of Debrecen, Departamento de Química \\ E-mail para contato: julianaalves035@gmail.com
}

\begin{abstract}
RESUMO - Este trabalho teve como objetivo caracterizar polióis industriais que são utilizados como matéria-prima na síntese de outros polímeros, como por exemplo o poliuretano. Para este fim foi utilizada a espectrometria de massa MALDI-TOF MS, uma técnica pouco explorada no Brasil na área de polímeros. A partir dos espectrogramas obtidos, foi possível ter conhecimento dos grupos laterais, composição química, índice de polidispersidade, distribuição de massas moleculares, entre outros fatores decisivos para uma caracterização precisa e eficiente.
\end{abstract}

\section{INTRODUÇÃO}

A área de Engenharia e Ciências dos Materiais, os polímeros em especial, têm constituído uma importante classe de materiais. Isso ocorre devido ao fato de que suas propriedades podem ser adaptadas a aplicações práticas específicas. Dessa forma, através da caracterização de polímeros por métodos analíticos precisos, pode-se ter o conhecimento da estrutura e composição química do polímero estudado, e consequentemente, o conhecimento de suas propriedades.

Lu et al. (2005) mostrou que dentre as técnicas de caracterização de polímeros, têm-se a Cromatografia de Permeação em Gel (GPC), também conhecida como Cromatografia por Exclusão de Tamanho (SEC) que é a frequentemente utilizada na caracterização, pois mede a massa molecular média e distribuição molecular do polímero. Porém, não é um método absoluto e pode apresentar imprecisões quando não há a presença de padrões para um determinado polímero. Assim, todos os métodos analíticos apresentam suas próprias limitações e o método de caracterização deve ser escolhido de acordo com as propriedades físico-químicas do polímero de interesse.

Entretanto, os trabalhos de Rader et al. (1998) mostrou que a técnica MALDI-TOF MS (Matrix-Assisted Laser Desorption/Ionization-Time Of Flight Mass Spectrometry) tem sido uma poderosa ferramenta na caracterização de compostos, pois determina a massa molecular absoluta independente da estrutura, com baixo consumo do analito e em curto tempo de análise. Esse método consiste na aplicação de uma carga na molécula ou átomo de interesse, e em seguida é medida a trajetória desses íons sob efeito da carga, no vácuo, a diferentes combinações de campo magnético e elétrico. 


\section{Congresso Brasileiro de Engenharia Química em Iniciação Científica \\ UFSCar - São Carlos - SP \\ 16 a 19 de Julho de 2017}

Através dessa técnica, é possível definir a massa molecular numérica média $(\mathrm{Mn})$ e a massa molecular ponderal média $(\mathrm{Mw})$ que são parâmetros de grande importância na caracterização de polímero. Segundo Mihail et al. (2005), Mn e Mw são funções do número de macromoléculas (Ni) e da massa molecular de cada cadeia (Mi) e podem ser calculados através das Equações (1) e (2) respectivamente.

$$
\begin{aligned}
& M n=\frac{\sum M i N i}{\sum N i} \\
& M w=\frac{\sum M i^{2} N i}{\sum M i N i}
\end{aligned}
$$

Outra característica importante do polímero é Índice de Polidispersidade (PDI) que é dado pela relação entre Mw e Mn, conforme mostrado na Equação (3).

$$
P D I=\frac{M w}{M n}
$$

Com o resultado obtido dessa relação, pode-se concluir se o polímero estudado apresenta cadeias com o mesmo comprimento (monodisperso) $\mathrm{PDI}=1$, ou se possui cadeias com comprimentos diferentes, polidisperso (PDI $>1$ ).

Através dos trabalhos de Aminlashgari et al. (2012) observou-se que a análise quantitativa em MALDI-TOF MS é considerada um método absoluto somente para uma distribuição de massas moleculares com baixa PDI. Valores menores que PDI $=1,1$ apresentam maior exatidão através dessa técnica. Porém para PDI $>1,1$ têm-se um aumento de desvios e os dados não são muito confiáveis, ou seja, essa é uma limitação do MALDITOF MS.

Essa nova técnica vem sendo explorada principalmente em moléculas com elevada massa molar como biomoléculas e inclusive a classe de polímeros sintéticos. Porém, até o momento, há poucos registros na literatura sobre essa técnica na área de polímeros no Brasil.

Dessa forma, o objetivo desse trabalho é utilizar essa técnica pouco difundida ainda no Brasil na caracterização de polímeros e formalizar um método de análise por MALDI-TOF MS para polióis com as mesmas características daqueles estudados neste trabalho. No caso, serão estudados polióis com diferentes massas molares por MALDI-TOF MS.

\section{MATERIAIS E MÉTODOS}

\subsection{Preparo das amostras}

Foram escolhidos 6 polióis comerciais diferentes com massa molecular média numérica teórica entre 800 a $5000 \mathrm{Da}$, conforme mostrado na Tabela 1. 


\section{Congresso Brasileiro de Engenharia Química em Iniciação Científica UFSCar - São Carlos - SP

Tabela 1 - Dados da ficha técnica de cada poliol estudado fornecido pelo próprio fabricante

\begin{tabular}{|c|c|c|c|c|c|c|}
\hline Nome comercial & $\begin{array}{c}\text { ROKOPOL } \\
\text { RF551 }\end{array}$ & PETOL 120-2 & $\begin{array}{c}\text { ROKOPOL } \\
\text { D2002 }\end{array}$ & PETOL 56-2 & PETOL 56-3 & ROKOPOL \\
M1170
\end{tabular}

Primeiramente, os polióis, NaTFA e DHB foram diluídos em metanol (pureza $\geq 99.8 \%$ ). Para isso, os poliois foram pesados separadamente em balança de laboratório com precisão de 0,01g, assim como o NaTFA e DHB, a fim de se obter soluções com concentração de $10 \mathrm{mg} / \mathrm{ml}$ de cada poliol em metanol, $20 \mathrm{mg} / \mathrm{ml}$ de NaTFA em metanol e $5 \mathrm{mg} / \mathrm{ml}$ de DHB em metanol.

Em seguida, a partir dessas soluções, foi preparada uma mistura com $10 \mu 1$ de poliól em metanol, $50 \mu 1$ de DHB em metanol e $5 \mu 1$ de NaTFA em metanol, essas misturas foram preparadas separadamente para cada poliol estudado, ou seja, no final foram obtidas 6 misturas diferentes. Depois transferiu-se com a pipeta volumétrica $0,5 \mu 1$ dessa mistura à placa de amostra do MALDI-TOF MS, deixou-se secar a mistura de cada amostra na placa de MALDI-TOF MS até se obter uma mistura homogênea sólida na placa.

\subsection{Análise em MALDI-TOF MS}

As análises foram feitas no MALDI-TOF MS modo reflector previamente calibrado com polietileno glicol (1540 Da), localizado no país Hungria, cidade Debrecen, Universidade de Debrecen, Departamento de química. Em seguida foi utilizado o software Bruker a fim de se obter os espectrogramas. Depois da análise concluída no MALDI-TOF MS, calcularam-se através do software Bruker o Mn, Mw e PDI de cada poliol com alta precisão, além disso determinou-se a unidade que se repete no polímero, o grau de polimerização, os grupos de ponta, ou seja, a composição do poliol.

\section{RESULTADOS E DISCUSSÃO}

Conforme descrito na metodologia, pôde-se calcular os valores de Mn, Mw e PDI para cada poliol. Tais dados podem ser encontrados na Tabela 2.

Tabela 2 - Mn teórica, Mn, Mw e PDI experimentais

\begin{tabular}{|c|c|c|c|c|c|c|}
\hline Nome comercial & $\begin{array}{c}\text { PETOL 120- } \\
2\end{array}$ & $\begin{array}{c}\text { ROKOPOL } \\
\text { RF551 }\end{array}$ & $\begin{array}{c}\text { ROKOPOL } \\
\text { D2002 }\end{array}$ & PETOL 56-2 & PETOL 56-3 & $\begin{array}{c}\text { ROKOPOL } \\
\text { M1170 }\end{array}$ \\
\hline Mn teórica (Da) & 1000 & 800 & 2000 & 2000 & 3000 & 5000 \\
\hline $\begin{array}{c}\text { Mn experimental } \\
(\mathrm{Da})\end{array}$ & 905 & 804 & 1982 & 1885 & 2977 & 4439 \\
\hline $\begin{array}{c}\text { Mw experimental } \\
(\mathrm{Da})\end{array}$ & 952 & 812 & 1998 & 1919 & 3008 & 4511 \\
\hline PDI experimental & 1,05 & 1,01 & 1,01 & 1,02 & 1,01 & 1,01 \\
\hline
\end{tabular}




\section{Congresso Brasileiro de Engenharia Química em Iniciação Científica \\ UFSCar - São Carlos - SP \\ 16 a 19 de Julho de 2017}

O PDI calculado está entre 1,01-1,05 (PDI < 1,1), indicando que a distribuição de massa molecular (MWD) de todos os polióis estudados é estreita e que, consequentemente, a exatidão da análise por MALDI-TOF MS é alta para tais polióis.

A Figura 1 apresenta o espectro do poliol Petol 120-2. A diferença entre um pico e outro é $58 \mathrm{Da}$ e conforme visto na Tabela 1, o Petol 120-2 é um PPG de alta pureza.

Figura 1 - O espectro MS do poliol Petol 120-2

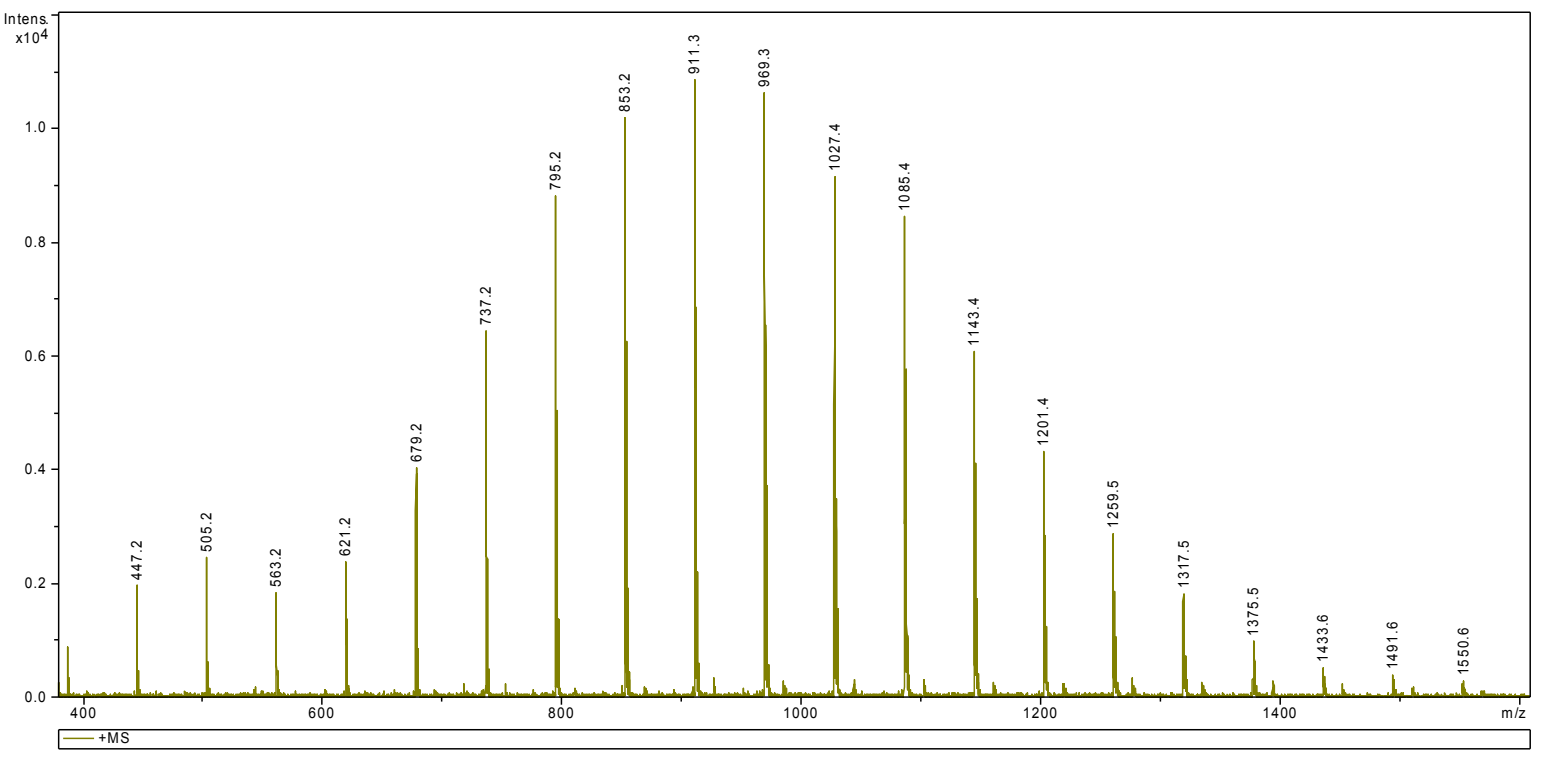

Cada pico no espectrograma representa um diferente grau de polimerização sendo observado um aumento de $58 \mathrm{Da}$ de um pico para o outro, o que corresponde à adição de um monômero de PPG à cadeia. A massa molar total do polímero é a massa da unidade que se repete multiplicada por $\mathrm{n}$, somada a massa molar do grupo de ponta. Isso equivale ao valor de $\mathrm{m} / \mathrm{z}$ do pico no espectro subtraído da massa molar do cátion $\mathrm{Na}^{+}(23 \mathrm{Da})$, conforme mostrado na Equação 4.

$\left(\mathrm{M}_{\mathrm{C} 3 \mathrm{H} 6 \mathrm{O}}\right) n+\mathrm{M}_{\text {grupo de ponta }}=\mathrm{m} / \mathrm{z}-\mathrm{M}_{\mathrm{Na}+}$

$58 \times \mathrm{n}+\mathrm{M}_{\text {grupo de ponta }}=(911,3-23)$

Quando $\mathrm{n}=15$

$\mathrm{M}_{\text {grupo de ponta }}=18 \mathrm{Da}$ (massa molar da $\mathrm{H}_{2} \mathrm{O}$ )

Para quaisquer valor de $\mathrm{n}>6$, a Mgrupo de ponta será o mesmo, para o Petol 120-2. Nesse caso o grupo de ponta é a água, e a fórmula química desse poliol é $\left[\left(\mathrm{C}_{3} \mathrm{H}_{6} \mathrm{O}\right)_{\mathrm{n}} \mathrm{H}_{2} \mathrm{O}+\mathrm{Na}\right]$ ${ }^{+}$. Os picos anteriores a $400 \mathrm{Da}(\mathrm{n}<6)$ não foram considerados uma vez que a série de polímeros está clara somente a partir de $400 \mathrm{Da}$.

A Figura 2 apresenta o espectro MS do Rokopol RF551, um polialquileno sorbitol que é um copolímero com a funcionalidade igual à de um óxido de propileno (PPG) e óxido de etileno (PEG) com o grupo sorbitol $\left(\mathrm{C}_{6} \mathrm{H}_{14} \mathrm{O}_{6}\right)$, conforme visto na Tabela 1. Nesse caso, com a ajuda de um programa chamado Isotope Pattern proveniente do software Bruker pôde-se identificar cada pico, porém somente alguns exemplos da composição química foram listados 
na Figura 2. Assim, a fórmula química para Rokopol RF551 é $\left[\left(\mathrm{C}_{2} \mathrm{H}_{4} \mathrm{O}\right)_{\mathrm{X}}\left(\mathrm{C}_{3} \mathrm{H}_{6} \mathrm{O}\right)_{\mathrm{Y}} \mathrm{C}_{6} \mathrm{H}_{14} \mathrm{O}_{6}+\right.$ $\mathrm{Na}]^{+}$sendo o grupo de ponta o sorbital, $\mathrm{C}_{6} \mathrm{H}_{14} \mathrm{O}_{6}$.

Figura 2 - O espectro MS do Rokopol RF551

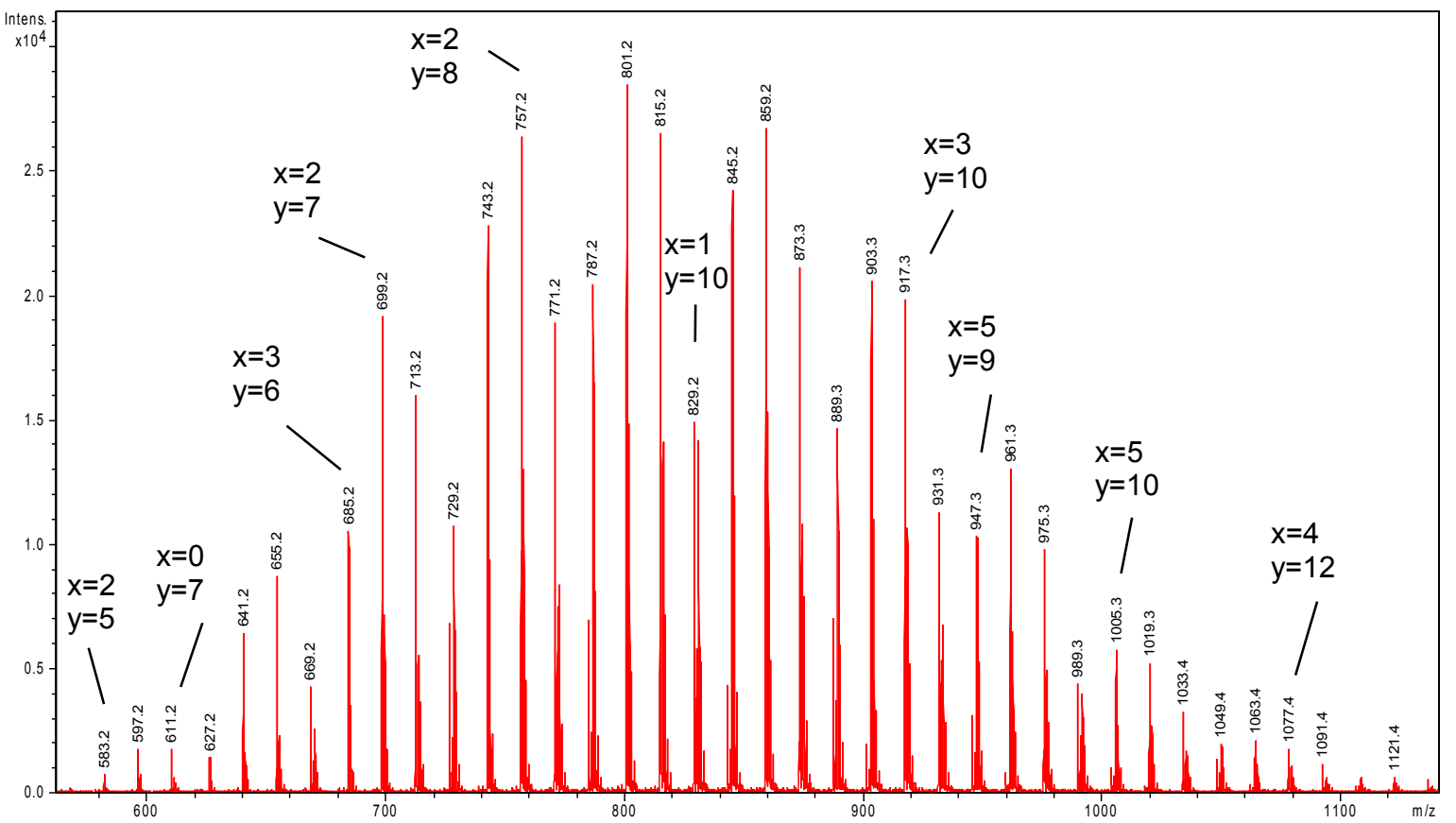

A Figura 3 apresenta o espectro MS do copolímero Rokopolo M1170. Nesse caso, o Mn teórico é muito elevado e o pico isotópico não pode ser separado no instrumento MALDITOF MS utilizado. Dessa forma não foi possível identificar a estrutura, e de modo geral, espectras de baixa resolução como essa são descartáveis.

Figura 3 - O espectro do poliol Rokopol M1170

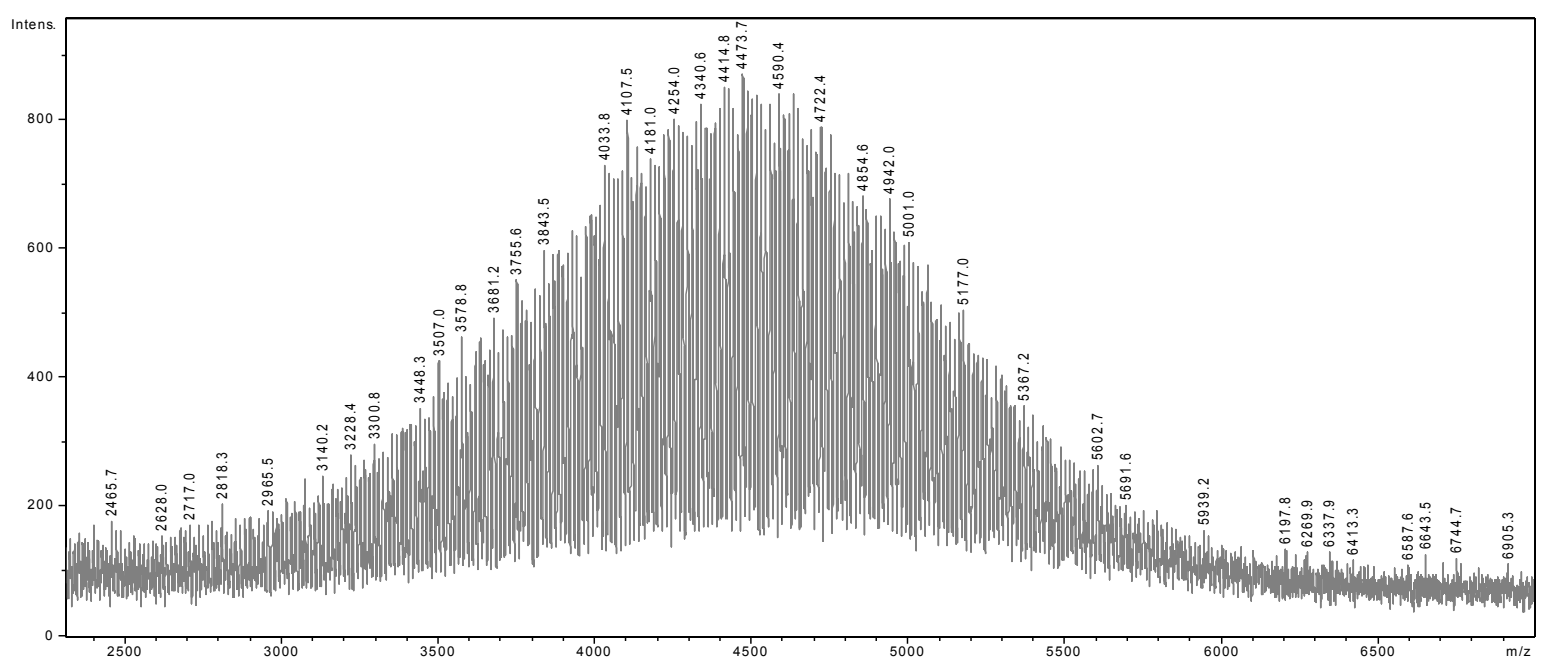

A Figura 4 apresenta 3 resoluções diferentes oriundas de pico isotópico (um único pico ampliado) do Rokopol RF551 (a), do Rokopol 2002 (b) e do Petol 56-3 (c). A Mn dessas amostras são 800, 2000 e 3000 respectivamente. 
Figura 4 - Os espectras MS do pico isotópico do (a) Rokopol RF551, (b) Rokopol 2002 and (c) Petol 56-3
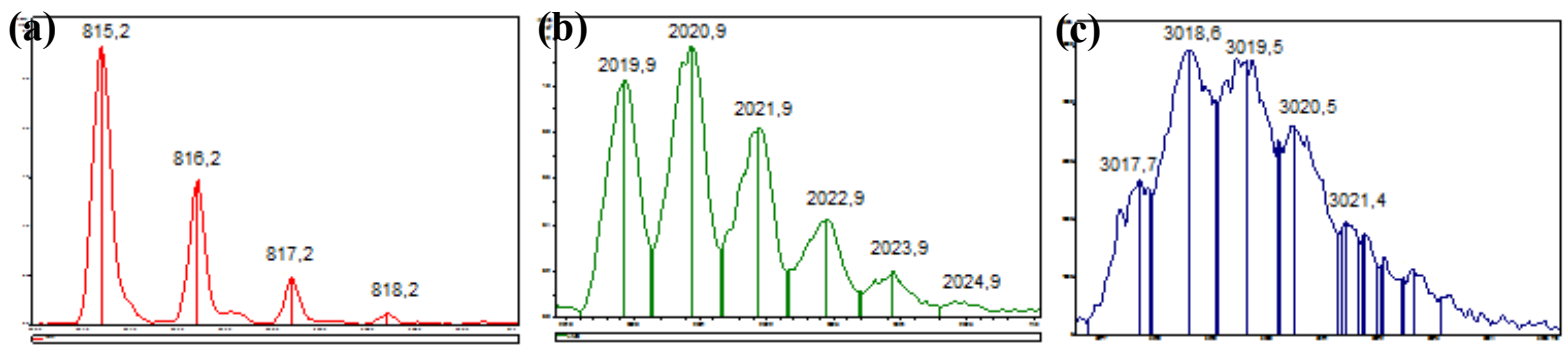

A partir da Figura 4 foi possível avaliar que a o pico isotópico na Figura 4a está perfeitamente separado e o espaçamento entre um pico e outro é significativamente grande e que não há presença de sobreposição de picos. Em contrapartida o pico isotópico da Figura $4 \mathrm{~b}$ tem uma Mn 2,5 vezes maior que Figura 4a e apresenta picos separados, porém não é uma separação perfeita. Por fim, a Figura $4 \mathrm{c}$ possui Mn 4 vezes maior que Figura $4 \mathrm{a}$ e apresenta uma baixa resolução e presença de sobreposição.

\section{CONCLUSÃO}

Baseando-se nos espectros obtidos pode-se concluir que todos os gráficos são típicos de espectro MS de polímeros por MALDI-TOF MS. Os valores de Mn, Mw e PDI experimentais foram calculados, e os valores Mn teórico e experimental se mostram bem próximos. Os valores de PDI estão na faixa 1,01-1,05 o que significa que tal metodologia é aplicável para esses polióis estudados.

Os espectros apresentam uma diminuição de resolução com o aumento da $\mathrm{Mn}$. A sobreposição de picos é mais frequente com o aumento da Mn quando se utiliza MALDI-TOF MS. Essas análises confirmam que o estudo de oligo-polios por MALDI-TOF MS é uma técnica de análise precisa, ou seja, $\mathrm{Mn}, \mathrm{Mw}$ e PDI podem ser medidos com precisão principalmente quando Mn está entre 800 a 2000Da. A partir de 3000Da o espectro tende a apresentar sobreposição e assim, perda de dados. Além disso, tal técnica é eficaz na caracterização de polímeros para síntese de outros polímeros.

\section{REFERENCIAS}

AMINLASHGARI N., HAKKARAINEN M. Emerging Mass Spectrometric Tools for Analysis of Polymers and Polymer Additives. Ed.; Springer, Berlin, 2012, Vol. 248, 137.

Lu X., Li Z. J., Chen G. Q. Polymer nanoparticles Ed. ELSEVIER ACADEMIC PRESS INC, San Diego, 2011, 299-323.

MIHAIL I. Chemistry and technology of polyols for polyurethanes. Ed. iSmithers Rapra, Reino Unido, 2005, 1-200.

RADER H. J. S. W., SCHREPP W. MALDI-TOF mass spectrometry in the analysis of synthetic polymers. Ed.; Acta Polymerica Alemanha, 1998, 19-204. 